\title{
Silicon-organic hybrid (SOH) devices and their use in comb-based communication systems
}

\author{
C. Koos $^{1,2, *}$, W. Freude ${ }^{1,2}$, L. Dalton ${ }^{5}$, T. J. Kippenberg ${ }^{3}$, L. P. Barry ${ }^{4}$, A. Ramdane ${ }^{6}$, F. Lelarge ${ }^{7}$, \\ S. Wolf ${ }^{1}$, H. Zwickel ${ }^{1,2}$, M. Lauermann ${ }^{1}$, C. Weimann ${ }^{1}$, W. Hartmann ${ }^{1,2}$, J. N. Kemal ${ }^{1}$, P. Marin ${ }^{1}$, P. Trocha ${ }^{1}$, J. Pfeifle ${ }^{1}$, \\ T. Herr ${ }^{3}$, V. Brasch ${ }^{3}$, R. T. Watts ${ }^{4}$, D. Elder ${ }^{5}$, A. Martinez ${ }^{6}$, V. Panapakkam ${ }^{6}$, N. Chimot ${ }^{7}$ \\ ${ }^{1}$ Institute of Photonics and Quantum Electronics (IPQ), Karlsruhe Institute of Technology (KIT), 76131 Karlsruhe, Germany \\ ${ }^{2}$ Institute of Microstructure Technology (IMT), Karlsruhe Institute of Technology (KIT), 76344 Eggenstein-Leopoldshafen, Germany \\ ${ }^{3}$ Ecole Polytechnique Fédérale de Lausanne (EPFL), 1015 Lausanne, Switzerland \\ ${ }^{4}$ The Rince Institute, School of Electronic Engineering, Dublin City University, Glasnevin, Dublin 9, Ireland \\ ${ }^{5}$ University of Washington, Seattle, WA 98195-1700, USA \\ ${ }^{6}$ Laboratoire de Photonique et Nanostructures, CNRS UPR20, Marcoussis, France \\ ${ }^{7}$ III-V Labs, Marcoussis, France \\ *email: christian.koos@kit.edu
}

\begin{abstract}
Advanced wavelength-division multiplexing (WDM) requires both efficient multi-wavelength light sources to generate optical carriers and highly scalable photonic-electronic interfaces to encode data on these carriers. In this paper, we give an overview on our recent progress regarding silicon-organic hybrid (SOH) integration and comb-based WDM transmission.
\end{abstract}

Keywords - Silicon photonics, silicon-organic hybrid (SOH) integration, frequency combs, optical interconnects

\section{SUMMARY}

High-speed optical interconnects within and between data centers rely on advanced wavelength-division multiplexing (WDM) schemes that are scalable to large channel counts. This requires power-efficient photonicelectronic interfaces that can be densely integrated at low cost as well as chip-scale light sources that can provide a multitude of narrowband optical carriers for WDM transmission. Photonic-electronic interfaces can be efficiently realized on the silicon photonic platform, in particular when exploiting the unique performance of organic electro-optic materials using the silicon-organic hybrid $(\mathrm{SOH})$ approach. At the same time, optical frequency combs lend themselves as multi-wavelength sources for scalable WDM transmission. This paper gives an overview on our recent progress in the fields of $\mathrm{SOH}$ integration and comb-based WDM transmission and discusses the prospects of merging the two approaches.

Silicon photonics shows tremendous potential for largescale photonic-electronic integration by fabless fabrication of photonic and electronic circuits [1]. Silicon as an optical material, however, falls short of properties that are indispensable for high-performance photonicelectronic interfaces. In particular, the inversion symmetry of the silicon crystal lattice inhibits technically relevant second-order nonlinearities, thereby making electro-optic modulators challenging. To overcome these deficiencies, the silicon-organic hybrid (SOH) concept combines silicon photonic circuits with highly efficient organic electro-optic materials [2]. This approach leads to highly efficient devices, featuring voltage-length products well below $1 \mathrm{Vmm}$ and energy consumptions of only a few fJ per bit [3]. The response of the electro-optic materials is ultra-fast and enables small-signal modulation at $100 \mathrm{GHz}$ [4], generation of $100 \mathrm{Gbit} / \mathrm{s}$ on-ofkeying (OOK) signals [5], and multi-level signaling at symbol rates of 64 GBd [6]. Moreover, we demonstrated generation of advanced modulation formats such as 16QAM at record-low energy consumptions and with symbol rates (bit rates) of up to $63 \mathrm{GBd}$ (252 Gbit/s) transmitted on a single wavelength and a single polarization [7] - [9]. We further show that the extraordinarily low operating voltage of $\mathrm{SOH}$ modulators allows operation of the devices directly from standard output ports of field-programmable gate arrays (FPGA), without the need for external amplifiers or digital-to-analog converters. Such schemes can be used even if higher-order modulation formats such as 16QAM are to be generated [10]. $\mathrm{SOH}$ approach is a versatile concept that goes far beyond electro-optic modulators. We have also shown that compact and power-efficient $\mathrm{SOH}$ phase shifters can be realized by using liquid crystals (LC) as cladding materials [11]. We have further demonstrated that the concept of $\mathrm{SOH}$ integration can be transferred to plasmonic waveguide structures, leading to plasmonic-organic hybrid $(\mathrm{POH})$ devices [12] that may open the route for modulation at $\mathrm{THz}$ frequencies [2].

Regarding WDM transmission based on optical frequency combs, our work aims at exploiting chip-scale comb sources that can be combined with $\mathrm{SOH}$ transmitters using monolithic or multi-chip integration concepts. Unlike carriers derived from a bank of individual laser modules, the tones of a comb are intrinsically equidistant in frequency, thereby enabling transmission at highest spectral efficiency. In addition, stochastic frequency variations of the carriers are strongly correlated, which enables effi-

(C) 2016 IEEE. Personal use of this material is permitted. Permission from IEEE must be obtained for all other uses, in any current or future media, including reprinting/republishing this material for advertising or promotional purposes, creating new collective works, for resale or redistribution to servers or lists, or reuse of any copyrighted component of this work in other works.

See http://www.ieee.org/publications_standards/publications/rights/index.html for more information. 
This article has been accepted for publication, but has not been fully edited. Content may change prior to final publication. Citation information: DOI https://doi.org/10.1109/GROUP4.2016.7739133

cient compensation of impairments caused by nonlinearities of the transmission fiber [13].

We performed a series of experiments to investigate the viability of chip-scale comb sources for WDM transmission. In a first set of experiments, we explore frequency comb generation using $\mathrm{SOH}$ electro-optic modulators, leading to line rates up to $1.152 \mathrm{Tbit} / \mathrm{s}$ on 9 optical carriers [14]. These devices enable large modulation depths at moderate drive voltages, thereby generating broadband frequency combs from a single continuous-wave (cw) laser line. Modulator-based comb generators lend themselves to monolithic co-integration with $\mathrm{SOH}$ transmitters. In a second set of experiments, we use gainswitching of an injection-locked laser diode to generate frequency combs [15]. These so-called gain-switched combs sources (GSCS) enable line rates of more than 2 Tbit/s using 24 comb lines as optical carriers. More recently, we have demonstrated that GSCS can not only act as light sources at the transmitter, but also as multiwavelength local oscillators at the receiver [16]. In a third set of experiments, we use quantum-dash modelocked laser diodes (MLLD) as frequency comb sources. These devices exhibit rather large optical linewidths, which either requires dedicated phase noise reduction schemes [17], self-homodyne detection [18], or digital phase tracking [19] to enable coherent communications with higher-order modulation formats at low symbol rates. At high symbol rates of, e.g., $40 \mathrm{GBd}$ or more, carrier phase noise is less detrimental, and no additional measures are needed. In a WDM experiment with 52 channels, we demonstrate transmission of an aggregate line rate of $8.32 \mathrm{Tbit} / \mathrm{s}$ over a transmission distance of $75 \mathrm{~km}$ [19]. A fourth set of experiments is dedicated to exploiting Kerr nonlinearities in integrated silicon-nitride (SiN) microcavities for frequency comb generation. We demonstrate coherent communication using a Kerr frequency comb source, achieving line rates of up to $1.44 \mathrm{Tbit} / \mathrm{s}$ on 20 carriers [20]. We are currently working on increasing the transmission speed to data rates beyond $10 \mathrm{Tbit} / \mathrm{s}$ by using cavity-soliton Kerr combs that can provide hundreds of spectral carriers [21], [22]. Our experiments show that frequency comb generation in chipscale devices represents a viable approach to terabit/s communications. GSCS, MLLD, and Kerr comb generators can be efficiently combined with $\mathrm{SOH}$ transmitters in optical multi-chip modules using the concept of photonic wire bonding [23], [24].

\section{ACKNOWLEDGEMENTS}

This work was supported by the European Research Council (ERC Starting Grant 'EnTeraPIC', number 280145), the Alfried Krupp von Bohlen und Halbach Foundation, the EU projects PhoxTroT and BigPipes, the BMBF project PHOIBOS, the Collaborative Research Centre "WavePhenomena" (CRC 1173) of Deutsche Forschungsgemeinschaft (DFG), the Helmholtz International Research School for Tera- tronics (HIRST), the Karlsruhe School of Optics \& Photonics (KSOP), and by the Karlsruhe Nano-Micro Facility (KNMF).

\section{REFERENCES}

[1] Sun, C. et al. 'Single-chip microprocessor that communicates directly using light" Nature 528, 534-538 (2015)

[2] Koos, C. et al. 'Silicon-Organic Hybrid (SOH) and PlasmonicOrganic Hybrid (POH) Integration', J. Lightw. Technol. 24, 256-268 (2016)

[3] Koeber, S. et al. 'Femtojoule electro-optic modulation using a silicon-organic hybrid device'; Light: Science \& Applications 4, e255 (2015)

[4] Alloatti, L. et al. '100 GHz silicon-organic hybrid modulator,' Light: Science \& Applications 3, e173 (2014)

[5] Hartmann, W. et al. '100 Gbit/s OOK using a silicon-organic hybrid (SOH) modulator,' 41th European Conf. Opt. Commun. (ECOC'15), Valencia, Spain, Sept. 27 - Oct. 5, 2015. Paper PDP1.4

[6] Lauermann, M. et al.: "Generation of 64 GBd 4ASK signals using a silicon-organic hybrid modulator at $80^{\circ} \mathrm{C}$ ”; Opt. Express 24, 9389-9396 (2016)

[7] Wolf, S. et al. 'An energy-efficient 252 Gbit/s silicon-based IQmodulator,' Optical Fiber Communication Conference (OFC'16), Los Angeles (CA), USA, March 20-24, 2016, Paper Th3J.2

[8] Lauermann, M et al.: "40 GBd 16QAM Signaling at $160 \mathrm{Gbit} / \mathrm{s}$ in a Silicon-Organic Hybrid (SOH) Modulator," , J. Lightw. Technol. 33, 1210-1216 (2015) (invited)

[9] Lauermann, M. et al. 'Low-power silicon-organic hybrid (SOH) modulators for advanced modulation formats,' Opt. Express 22, 29927-29936 (2014)

[10] Wolf, S. et al. "DAC-less Amplifier-less Generation and Transmission of QAM Signals Using Sub-Volt Silicon-organic Hybrid Modulators," J. Lightw. Technol. 33, 1425-1432 (2015)

[11] Pfeifle, J. et al. 'Silicon-organic hybrid phase shifter based on a slot waveguide with a liquid-crystal cladding,' Opt. Express 20 (2012), 15359-15376

[12] Melikyan, A. et al. 'Plasmonic-organic hybrid (POH) modulators for OOK and BPSK signaling at $40 \mathrm{Gbit} / \mathrm{s}$,' Opt. Express 23, 9938-9946 (2015)

[13] Temprana, E. et al. "Overcoming Kerr-induced capacity limit in optical fiber transmission.” Science 348, 1445-1448 (2015).

[14] Weimann, C. et al.: 'Silicon-organic hybrid (SOH) frequency comb sources for terabit/s data transmission,' Opt. Express 22, 3629-3637 (2014).

[15] Pfeifle, J. et al. "Flexible terabit/s Nyquist-WDM superchannels using a gain-switched comb source.” Opt. Express 23, 724 - 738 (2015)

[16] Kemal, J. N. et al. "Parallel multi-wavelength intradyne reception using an optical frequency comb as a local oscillator." 41th European Conf. Opt. Commun. (ECOC'15), Valencia, Spain, Sept. 27 - Oct. 5, 2015. Paper P.4.18

[17] Pfeifle, J. et al. "Simultaneous phase noise reduction of 30 comb lines from a quantum-dash mode-locked laser diode enabling coherent Tbit/s data transmission.” Optical Fiber Communication Conference (OFC'15), Los Angeles (CA), USA, 22 - 26 March , Paper Tu3I.5. (2015)

[18] Pfeifle, J. et al. "Coherent terabit communications using a quantum-dash mode-locked laser and self-homodyne detection." Optical Fiber Communication Conference (OFC'15), Los Angeles (CA), USA, 22 - 26 March, Paper W2A.19. (2015)

[19] Marin, P. et al. "8.32 Tbit/s coherent transmission using a quantum-dash mode-locked laser diode.” Conf. on Lasers and Electro-Optics (CLEO'16), San Jose (CA), USA, June 5-10, 2016. Paper STh1F.1

(C) 2016 IEEE. Personal use of this material is permitted. Permission from IEEE must be obtained for all other uses, in any current or future media, including reprinting/republishing this material for advertising or promotional purposes, creating new collective works, for resale or redistribution to servers or lists, or reuse of any copyrighted component of this work in other works.

See http://www.ieee.org/publications_standards/publications/rights/index.html for more information. 
This article has been accepted for publication, but has not been fully edited. Content may change prior to final publication.

Citation information: DOI https://doi.org/10.1109/GROUP4.2016.7739133

[20] Pfeifle, J. et al. "Coherent terabit communications with microresonator Kerr frequency combs.” Nature Photon. 8, 375380 (2014)

[21] Pfeifle, J. et al. "Full C and L-band transmission at 20 Tbit/s using cavity-soliton Kerr frequency combs.” Conf. on Lasers and Electro-Optics (CLEO'15), San Jose (CA), USA, May 1015, 2015. Paper STh5C.8

[22] Brasch et al., "Photonic chip-based optical frequency comb using soliton-induced Cherenkov radiation.” Science 22, 357360 (2016)
[23] Lindenmann, N. et al.: 'Photonic wire bonding: a novel concept for chip-scale interconnects,' Opt. Express 20, 17667-17677 (2012)

[24] Lindenmann, N.; et al. 'Connecting silicon photonic circuits to multicore fibers by photonic wire bonding;' J. Lightw. Technol. 33, 755-760 (2015)

(C) 2016 IEEE. Personal use of this material is permitted. Permission from IEEE must be obtained for all other uses, in any current or future media, including reprinting/republishing this material for advertising or promotional purposes, creating new collective works, for resale or redistribution to servers or lists, or reuse of any copyrighted component of this work in other works. See http://www.ieee.org/publications_standards/publications/rights/index.html for more information. 